\title{
ANÁLISE DOS LAUDOS DE PAPANICOLAOU REALIZADOS EM UMA UNIDADE BÁSICA DE SAÚDE
}

Ana Paula Vieira Bringel'1, Marina Pessoa de Farias Rodrigues², Eglídia Carla Figueirêdo Vidal ${ }^{3}$

\begin{abstract}
RESUMO: Estudo descritivo teve por objetivo caracterizar os aspectos microbiológicos e epiteliais presentes nos exames preventivos realizados em uma unidade básica de saúde de Juazeiro do Norte - Ceará. Os dados foram coletados de julho a novembro de 2011, mediante a análise de 782 resultados de Papanicolaou realizados entre março de 2008 e outubro de 2011 e que constavam no livro de registros de preventivos da unidade. Os dados foram analisados com auxílio do programa estatístico SPSS 16.0. Os resultados evidenciaram maior procura de mulheres entre 25 a 34 anos $(28,4 \%)$ para a realização do exame; no esfregaço houve prevalência de epitélio escamoso $(80,9 \%)$, Cocos $(49,2 \%)$ e agentes infecciosos como Gardnerella vaginalis (65\%), Cândida sp (23,7\%) e Trichomonas vaginalis (5,6\%). O profissional de saúde precisa identificar os fatores de risco associados às afecções, possibilitando prevenção do câncer do colo uterino e controle das Doenças Sexualmente Transmissíveis. PALAVRAS- CHAVE: Saúde da mulher; Esfregaço vaginal; Câncer do colo do útero.

\section{ANALYSIS OF PAPANICOLAOU TEST RESULTS CARRIED OUT IN A PRIMARY HEALTH CENTER}

\begin{abstract}
This descriptive study aimed to characterize the microbiological and epithelial aspects present in the screening exams carried out in a primary health center in Juazeiro do Norte - Ceará. The data was collected from July to November 2011, through the analysis of the results of 782 smear tests undertaken between March 2008 and October 2011, included in the center's record-book for screening tests. The data was analyzed with the help of the statistical program SPSS version 16.0. The results evidenced a greater demand among women between 25 and 34 years old (28.4\%) for the test; in the swab specimen there was a prevalence of squamous epithelium (80.9\%), coccus (49.2\%) and infectious agents such as Gardnerella vaginalis (65\%), Cândida sp (23.7\%) and Trichomonas vaginalis (5.6\%). The health professional needs to identify the risk factors associated with the conditions, making it possible to prevent cervical cancer and control Sexually Transmitted Diseases. KEYWORDS: Women's health; Vaginal swab specimen; Cervical cancer.

\section{ANÁLISIS DE LOS LAUDOS DE PAPANICOLAOU REALIZADOS EN UNA UNIDAD BÁSICA DE SALUD}

RESUMEN: Estudio descriptivo que tuvo por finalidad caracterizar los aspectos microbiológicos y epiteliales presentes en los exámenes preventivos realizados en una unidad básica de salud de Juazeiro do Norte - Ceará. Los datos fueron obtenidos de julio a noviembre de 2011, por medio de análisis de 782 resultados de Papanicolaou realizados entre marzo de 2008 y octubre de 2011 y que estaban en el libro de registros de preventivos de la unidad. Los datos fueron analizados con ayuda del programa estadístico SPSS 16.0. Los resultados evidenciaron mayor búsqueda por mujeres entre 25 y 34 años $(28,4 \%)$ para realización del examen; en la colecta hubo prevalencia de epitelio escamoso $(80,9 \%)$, Cocos $(49,2 \%)$ y agentes infecciosos como Gardnerella vaginalis (65\%), Candida sp (23,7\%) y Trichomonas vaginalis (5,6\%). El profesional de salud debe identificar los factores de riesgo asociados a las afecciones, posibilitando prevención del cáncer de cuello de útero y control de las Enfermedades Sexualmente Transmisibles.

PALABRAS CLAVE: Salud de la mujer; Examen; Cáncer de cuello del útero.

\footnotetext{
${ }^{1}$ Acadêmica de Enfermagem da Universidade Regional do Cariri - URCA. Bolsista PIBIC-URCA. Membro do Grupo de Pesquisa em Saúde Coletiva - GRUPESC.

${ }^{2}$ Enfermeira. Especialista em Assistência e Gestão em Saúde da Família. Membro do GRUPESC.

${ }^{3}$ Enfermeira. Mestre em Enfermagem. Professora do Departamento de Enfermagem da URCA. Membro do GRUPESC.
}

Autor correspondente:

Recebido: $18 / 06 / 2012$

Ana Paula Vieira Bringel Aprovado: 20/08/2012

Universidade Regional do Cariri

Rua José Marconi Alencar, 47 - 63030-590 - Juazeiro do Norte-CE-Brasil

E-mail: anapaulabringel@yahoo.com 


\section{INTRODUÇÃO}

O câncer do colo uterino ou câncer cervical consiste na replicação desordenada do epitélio de revestimento do órgão, comprometendo o tecido subjacente e podendo alastrar-se por órgãos adjacentes ou distantes ${ }^{(1)}$. Este caracteriza-se como o terceiro tipo de câncer que mais acomete as mulheres, com cerca de 530 mil casos novos por ano no mundo, sendo responsável pela morte de aproximadamente 275 mil mulheres anualmente ${ }^{(2)}$. No Brasil, em 2012, são esperados 17.540 casos novos, com um risco estimado de 17 casos a cada 100 mil mulheres ${ }^{(3)}$.

Em face da epidemiologia, o câncer do colo uterino constitui importante problema de saúde pública, previsto na Agenda Política de Saúde do Brasil, entre as seis prioridades do Pacto pela Vida do Sistema Único de Saúde (SUS). A neoplasia tem início com transformações intraepiteliais progressivas que podem evoluir para um processo invasor num período que varia de 10 a 20 anos. Graças a essa lenta progressão é possível a detecção precoce de lesões pré-malignas ou malignas e a implantação de tratamento ${ }^{(4)}$.

Nesse contexto, as transformações celulares anteriores ao processo neoplásico merecem acompanhamento e seguimento especial no atendimento da mulher. Os epitélios do colo uterino sofrem diferenciação ao longo de sua vida reprodutiva, provocando metaplasia e constituindo uma zona de transformação que, associada à maturação da célula do epitélio em transformação e motivada pelo Papilomavírus Humano (HPV), pode evoluir para um processo celular neoplásico benigno e, consequentemente, para a malignidade ${ }^{(5)}$.

Diante disso, o Ministério da Saúde preconiza, como estratégia prioritária de prevenção desse agravo, em mulheres de 25 a 59 anos, a realização do exame de Papanicolaou, com a implementação desta prática no cotidiano de trabalho das equipes da Estratégia Saúde da Família (ESF) ${ }^{(1,3)}$. No Brasil, o estímulo à realização desse exame surgiu com a implantação do Programa de Atenção Integral à Saúde da Mulher, em 1989, como procedimento de consulta ginecológica, focado na atenção primária à saúde ${ }^{(1)}$.

O exame de Papanicolaou, técnica amplamente difundida há mais de $40 \operatorname{anos}^{(6)}$, consiste na coleta e análise das células oriundas da ectocérvice e da endocérvice, resultantes da raspagem do colo do útero, exposto pela introdução do espéculo vaginal e decorrente da consulta ginecológica de rotina, com a finalidade de verificar a existência de alterações pré-malignas ou malignas em suas células, possibilitando a detecção precoce e a realização do tratamento oportuno ${ }^{(7)}$.

Além disso, pode-se a partir desse exame detectar sinais de inflamações da vulva e da vagina, favorecendo o rastreamento e detecção de vulvovaginites. Essas afecções caracterizam-se clinicamente por aumento do conteúdo vaginal acompanhado de odor desagradável, que piora no período menstrual e após a relação sexual ${ }^{(8)}$. Não obstante, além do desconforto que causa nas pacientes, existem alguns agentes patogênicos que se enquadram no grupo das doenças sexualmente transmissíveis, podendo ser considerado um problema de saúde pública ${ }^{(9)}$.

Consubstancialmente, resultados de pesquisas evidenciaram que nos municípios de Paranavaí - Paraná e Goiânia - Goiás, entre as infecções vaginais mais frequentes estão candídiase, vaginose bacteriana e trichomoníase, principalmente em mulheres em idade reprodutiva ${ }^{(10-11)}$. As doenças sexualmente transmissíveis (DST) e o câncer de colo de útero também se enquadram entre as doenças que promovem a maior procura das mulheres pelos serviços de saúde. Estudos de prevalência evidenciaram que mulheres com história, ou portadoras de DST, apresentam maior risco para o câncer cérvicouterino para outros fatores que aumentam esse risco, como a infecção pelo papiloma vírus humano (HPV) ${ }^{(1)}$.

Diante do exposto, a partir da vivência particular das autoras em Unidade Básica de Saúde (UBS), observa-se elevado número de laudos citopatológicos com alguma alteração fisiológica, cujo cenário chama a atenção e incita o pensamento crítico para a associação entre a realização de exame cérvicouterino e a presença de alterações microbiológicas e epiteliais.

Além disso, evidencia-se a necessidade da realização de pesquisa com essa temática, para nortear e embasar cientificamente outras propostas de intervenção efetivas no âmbito da prevenção do câncer do colo uterino, das DST e das infecções vaginais. Ainda, pelo fato de existir poucos estudos nos bancos de dados de saúde sobre o tema em questão.

Assim, este estudo teve por objetivo caracterizar os aspectos microbiológicos e epiteliais dos resultados de exames preventivos de câncer do colo uterino realizados em uma unidade básica de saúde de Juazeiro do Norte, Estado do Ceará. 


\section{MÉTODO}

Trata-se de um estudo do tipo descritivo, retrospectivo e transversal, alcançado por meio de pesquisa documental com abordagem quantitativa. Ressalta-se que os documentos constituem fonte rica e estável de dados além de subsistirem ao longo do tempo, tornando-se a mais importante fonte de dados em pesquisa de natureza histórica ${ }^{(12)}$.

O local da realização do estudo foi a UBS 55, sede da ESF 55, no Município de Juazeiro do Norte. A escolha desse deu-se mediada pelos seguintes critérios: acessibilidade das pesquisadoras, boa organização do serviço, registro adequado dos dados dos laudos, possibilitando a fiel coleta dos dados, e observações realizadas em práticas de estágios supervisionados do Curso de Graduação em Enfermagem da Universidade Regional do Cariri.

O estudo utilizou como fonte de captação dos dados os registros dos exames de prevenção de câncer do colo uterino realizados na citada UBS, presentes em um livro destinado às anotações dos atendimentos ginecológicos para realização do exame de Papanicolaou. Nesse, constam o nome completo e o endereço das mulheres, o resultado do exame, observações adicionais e o tipo de encaminhamento, quando o mesmo torna-se indispensável, possibilitando ainda, a realização de busca ativa e acompanhamento do atendimento, quando estes apresentam-se necessários.

O universo da pesquisa alcançou $100 \%$ dos registros dos resultados contidos no citado livro, e refere-se ao período de março de 2008, ano no qual a UBS entrou em funcionamento, até outubro de 2011. Totalizaram 782 registros de laudos citopatológicos, que correspondem a 42 meses de atendimento.
Os dados foram coletados no período de julho a novembro de 2011, com emprego de uma planilha destinada ao registro e organização dos dados desenvolvida para este estudo, tendo como variáveis: número de atendimento, aspectos microbiológicos e respectiva relação dos agentes infecciosos à sugestão de vulvoginite, e achados epiteliais, todos relacionados à distribuição etária das mulheres. Os resultados obtidos foram submetidos ao programa estatístico SPSS 16.0 e apresentados em números absolutos e percentuais.

O projeto de pesquisa foi aprovado pelo Parecer n. 22/2010, emitido pelo Comitê de Ética em Pesquisa da Universidade Regional do Cariri, obedecendo-se às normas da Resolução n. 196/96, do Conselho Nacional de Saúde.

\section{RESULTADOS}

\section{Perfil etário de mulheres submetidas ao exame de Papanicolaou}

A tabela 1 mostra que, ao longo dos quase quatro anos (2008-2011) de atendimento ginecológico às mulheres vinculadas `a UBS, a faixa etária prevalente das submetidas ao exame de Papanicolaou foi de 25 a 34 anos (28,4\%), seguida daquelas com 35 a 44 anos (24\%) e de 15 a 24 anos (23\%), totalizando $75,4 \%$ de mulheres entre 15 e 44 anos.

A realização do exame preventivo demonstrou decrescer com o aumento da idade da mulher, conforme se pode notar pela diminuição do número de atendimentos a partir da faixa etária de 45 a 54 anos $(12,3 \%)$, 55 a 64 anos $(7,6 \%)$ e acima de 65 anos (4,3\%).

Ademais, foram evidenciados três resultados de exame em faixa etária inferior a 15 anos, caracterizando 0,4\% dos atendimentos, nos anos de 2008 e 2011.

Tabela 1 - Distribuição dos exames de Papanicolaou de acordo com a faixa etária e o ano de realização. Juazeiro do Norte, 2008 a 2011

\begin{tabular}{|c|c|c|c|c|c|c|}
\hline \multirow[t]{3}{*}{ Faixa Etária } & \multicolumn{4}{|c|}{ Anos } & \multicolumn{2}{|c|}{ Total } \\
\hline & 2008 & 2009 & 2010 & 2011 & & \\
\hline & $\mathrm{N}(\%)$ & $\mathrm{N}(\%)$ & $\mathrm{N}(\%)$ & $\mathrm{N}(\%)$ & $\mathrm{N}$ & $\%$ \\
\hline$<15$ & $01(0,6)$ & - & - & $02(1,7)$ & 03 & 0,4 \\
\hline $15-24$ & $53(29,4)$ & $51(20,2)$ & $52(22,2)$ & $24(20,9)$ & 180 & 23,0 \\
\hline $25-34$ & $54(30,0)$ & $83(32,8)$ & $57(24,4)$ & $28(24,3)$ & 222 & 28,4 \\
\hline $35-44$ & $35(19,4)$ & $65(25,7)$ & $57(24,4)$ & $31(27,0)$ & 188 & 24,0 \\
\hline $45-54$ & $21(11,7)$ & $31(12,2)$ & $30(12,8)$ & $14(12,2)$ & 96 & 12,3 \\
\hline $55-64$ & $11(6,1)$ & $14(5,5)$ & $21(8,9)$ & $13(11,3)$ & 59 & 7,5 \\
\hline$>65$ & $05(2,8)$ & $09(3,6)$ & $17(7,3)$ & $03(2,6)$ & 34 & 4,4 \\
\hline Total & $180(100)$ & $253(100)$ & $234(100)$ & $115(100)$ & $782(100)$ & $100(100)$ \\
\hline
\end{tabular}




\section{Aspectos microbiológicos nos registros dos exames de Papanicolaou}

Verificou-se que, dentre os 782 (100\%) laudos citopatológicos registrados, 699 (89,4\%) mostraram alguma alteração microbiológica. Entretanto, observa-se na tabela 2 que $\operatorname{Cocos}(\mathrm{n}=344)$ apresentaram maior ocorrência, correspondendo a 49,21\% dos registros. Seguiram-se os Bacilos que demonstraram uma ocorrência de 291 registros (41,6\%). Lactobacillus sp., embora sejam caracterizados como microbiota fisiológica prevalente na região genital feminina, foram menos prevalentes, correspondendo a 64 registros $(9,2 \%)$, e com menor prevalência entre resultados de mulheres maiores de 65 anos de idade (1,6\%).

Assim, evidenciamos Cocos com maior achado nas faixas etárias de 25 a 34 anos $(29,4 \%)$ e de 15 a 24 anos (21,8\%) e menor evidência naquelas com menos de 15 anos $(0,3 \%)$. Lactobacillus sp teve menor achado nas mulheres com mais de 65 anos de idade (1,6\%), concentrado na faixa etária de 25 a 34 anos (32,8\%) e 35 a 44 anos (34,4\%). Já Bacilos teve predominância em mulheres entre 15 a 24 anos $(32,3 \%)$ e 25 a 34 anos $(31,3 \%)$, com menor achado naquelas com menos de 15 anos $(0,7 \%)$ e entre 55 e 64 anos

Tabela 2 - Distribuição da microbiota identificada em exame Papanicolaou segundo a faixa etária das mulheres. Juazeiro do Norte, 2008 a 2011

\begin{tabular}{lcccccc}
\hline $\begin{array}{c}\text { Microbita } \\
\text { vaginal }\end{array}$ & \multicolumn{2}{c}{ Cocos } & \multicolumn{2}{c}{ Bacilos } & \multicolumn{2}{c}{ Lactobacillus sp } \\
$\begin{array}{l}\text { Faixa } \\
\text { etária }\end{array}$ & $\mathrm{N}$ & $\%$ & $\mathrm{~N}$ & $\%$ & $\mathrm{~N}$ & $\%$ \\
\hline$<15$ & & & & & - & \\
$15-24$ & 01 & 0,3 & 02 & 0,7 & 13 & 20,3 \\
$25-34$ & 15 & 21,8 & 94 & 32,3 & 21 & 32,8 \\
$35-44$ & 71 & 29,4 & 91 & 31,3 & 22 & 34,4 \\
$45-54$ & 45 & 20,6 & 63 & 21,6 & 05 & 7,8 \\
$55-64$ & 37 & 13,1 & 31 & 10,7 & 02 & 3,1 \\
$>65$ & 14 & 4,1 & 02 & 0,7 & 01 & 1,6 \\
Total $*$ & 344 & 100 & 291 & 2,7 & 64 & 100 \\
\hline
\end{tabular}

*Os dados desta tabela referem-se aos 699 laudos em que foram encontradas alterações microbiológicas.

$(0,7 \%)$.

A microbiota vaginal identificada nos laudos de exames de Papanicolaou possibilitou estabelecer a ocorrência de vulvovaginite, relacionando-se os agentes etiológicos mais evidenciados à faixa etária das mulheres (Tabela 3). Não obstante, dentre os 782 (100\%) laudos citopatológicos registrados, 160 (20,5\%) apresentaram alteração em relação à presença de algum agente infeccioso.

Os resultados sugestivos de Gardnerella vaginalis corresponderam a 104 (65\%) do total dos registros com alterações, tendo maior incidência $(33,7 \%)$ na faixa etária de 25 a 34 anos, e um decréscimo inversamente proporcional à idade. Cândida sp esteve presente em $38(23,7 \%)$ laudos, com prevalência acentuada na faixa

Tabela 3 - Distribuição dos agentes infecciosos presentes em exame de Papanicolaou segundo a faixa etária. Juazeiro do Norte, 2008 a 2011

\begin{tabular}{|c|c|c|c|c|c|c|c|c|c|c|}
\hline \multirow{2}{*}{ Faixa etária } & \multicolumn{2}{|c|}{ Candida sp } & \multicolumn{2}{|c|}{$\begin{array}{l}\text { Gardnerella - } \\
\text { Mobiluncus }\end{array}$} & \multicolumn{2}{|c|}{$\begin{array}{l}\text { Trichomonas } \\
\text { vaginalis }\end{array}$} & \multicolumn{2}{|c|}{ Outros** } & \multicolumn{2}{|c|}{ TOTAL } \\
\hline & $\mathrm{N}$ & $\%$ & $\mathrm{~N}$ & $\%$ & $\mathrm{~N}$ & $\%$ & $\mathrm{~N}$ & $\%$ & $\mathrm{~N}$ & $\%$ \\
\hline $15-24$ & 13 & 34,2 & 33 & 31,7 & 01 & 11,1 & 04 & 44,5 & 51 & 31,9 \\
\hline $25-34$ & 10 & 26,3 & 35 & 33,7 & 02 & 22,2 & 02 & 22,2 & 49 & 30,6 \\
\hline $35-44$ & 09 & 23,7 & 18 & 17,3 & 03 & 33,4 & - & - & 30 & 18,8 \\
\hline $45-54$ & 04 & 10,5 & 10 & 9,6 & 02 & 22,2 & 02 & 22,2 & 18 & 11,2 \\
\hline $55-64$ & 02 & 5,3 & 05 & 4,8 & 01 & 11,1 & 01 & 11,1 & 09 & 5,6 \\
\hline$>65$ & - & - & 03 & 2,9 & - & - & - & - & 03 & 1,9 \\
\hline Total* & 38 & 100 & 104 & 100 & 09 & 100 & 09 & 100 & 160 & 100 \\
\hline
\end{tabular}

*Os dados desta tabela referem-se aos 160 laudos em que foram encontrados agentes infecciosos. **Actinomyces sp; Herpes; Microbiota mista. 
etária de 15 a 24 anos (34,2\%) e ausência de registro na faixa etária acima de 65 anos. Trichomonas vaginalis foi identificado em 9 exames (5,6\%) entre os 160 laudos de exames Papanicolaou alterados. Evidenciou-se, também a presença de outros microrganismos como Actinomyces $s p$, do tipo Herpes e microbiota mista, porém sua representatividade foi pequena e foram agrupados em uma mesma categoria, registrada como Outros $(\mathrm{n}=09 ; 5,6 \%)$.

\section{Aspectos epiteliais nos registros dos exames de Papanicolaou}

Quanto ao tipo de epitélio presente nos laudos das amostras coletadas (Tabela 4) não se evidenciou a presença de ambos os tipos de epitélios em todos os registros dos laudos: prevaleceu o epitélio escamoso, que correspondeu a 633 casos $(80,9 \%)$ entre os 782 registros. Este foi seguido do epitélio glandular com 384 casos (49,1\%), e do epitélio metaplásico, com 58 registros (7,4\%).

Esta variável permitia mais de uma resposta e, ao analisar os laudos, foi tratada isolando-se cada epitélio. Dessa forma, o total de epitélio não coincide com o total de laudos. Destaca-se as seguintes ocorrências: o epitélio escamoso em número maior que os outros epitélios nas faixas etárias de 25 a 34 anos (29,8\%), e 35 a 44 anos (25,3\%); o epitélio glandular apresentou-se mais prevalente nas faixas etárias de 25 a 34 anos $(29,9 \%)$ e de 35 a 44 anos (28,4\%). Já o epitélio metaplásico, foi predominante de 35 a 44 anos $(27,6 \%)$ e de 25 a 34 anos $(25,9 \%)$.

Contudo, considera-se a necessidade de ser satisfatório para análise o esfregaço que conta com, no mínimo dois epitélios presentes, condição essencial à emissão de um laudo citopatológico do colo uterino, cujo registro foi apreciado neste estudo.

\section{DISCUSSÃO}

Os resultados revelaram predomínio de mulheres submetidas ao exame de Papanicolaou com idade inferior a 35 anos, em semelhança a estudo realizado na cidade de São Paulo que evidenciou que mulheres na faixa etária de 25 a 34 anos apresentam-se mais constantes na cobertura dos exames de Papanicolaou ${ }^{(5)}$.

Tabela 4 - Distribuição do epitélio registrado em relação à faixa etária nos exames de Papanicolaou. Juazeiro do Norte, 2008 a 2011

\begin{tabular}{|c|c|c|c|c|c|c|}
\hline \multirow{2}{*}{$\begin{array}{l}\text { Epitélio } \\
\text { Faixá } \\
\text { etária }\end{array}$} & \multicolumn{2}{|c|}{ Escamoso } & \multicolumn{2}{|c|}{ Glandular } & \multicolumn{2}{|c|}{ Metaplásico } \\
\hline & $\mathrm{N}$ & $\%$ & $\mathrm{~N}$ & $\%$ & $\mathrm{~N}$ & $\%$ \\
\hline $15-24$ & 132 & 20,9 & 77 & 20,1 & 10 & 17,2 \\
\hline $25-34$ & 189 & 29,8 & 115 & 29,9 & 15 & 25,9 \\
\hline $35-44$ & 160 & 25,3 & 109 & 28,4 & 16 & 27,6 \\
\hline $45-54$ & 76 & 12,0 & 44 & 11,5 & 11 & 18,9 \\
\hline $55-64$ & 48 & 7,6 & 25 & 6,5 & 03 & 5,2 \\
\hline$>65$ & 28 & 4,4 & 14 & 3,6 & 03 & 5,2 \\
\hline TOTAL* & 633 & 100 & 384 & 100 & 58 & 100 \\
\hline
\end{tabular}

*Total da tabela maior que a amostra de exames analisados. Presença de mais de um epitélio no mesmo exame.

Mulheres com idade superior a 55 anos foram as que menos se submeteram ao exame de Papanicolaou, contrapondo-se às evidências de prevalência de câncer do colo uterino naquelas com mais de 50 anos e com elevação dessa prevalência proporcional ao aumento da idade. Ademais, mulheres com 60 anos ou mais possuem maior probabilidade de desenvolver o câncer de colo uterino ${ }^{(13)}$.

Consubstancialmente, estudo conduzido nos Estados Unidos, revelou que as proporções de exames de citologia oncótica alterados foram maiores nas faixas etárias mais jovens, porém as taxas de câncer invasivo aumentaram com a idade, atingindo valores máximos entre 50 e 64 $\operatorname{anos}^{(14)}$. Desta forma, com vistas à prevenção do câncer de colo uterino, com detecção de lesões precursoras, evidencia-se a necessidade de atenção especial às mulheres em todas as faixas etárias, a partir do início sexual, mas reconhecendo a devida importância de cuidar daquelas que ultrapassam o período fértil.

Nesse estudo, a microbiota detectada nos laudos de exames de Papanicolaou foi representada por elevada prevalência de Cocos e menor de Lactobacillus. Entretanto, Cocos e Bacilos fazem parte da microbiota vaginal e não se caracterizam como infecções que necessitam de tratamento. 
Neste caso, a conduta clínica depende da sintomatologia, e a cliente deve ser encaminhada para avaliação ginecológica quando houver necessidade ${ }^{(1)}$. Por outro lado, Lactobacillus $s p$ é a espécie dominante na microbiota vaginal; estudos recentes apontam que, em algumas mulheres, microbiota vaginal não dominada por esse microrganismo pode ser normal e não necessariamente patogênica ${ }^{(15)}$.

No tocante aos agentes infecciosos identificados, Cândida sp e Gardnerella/Mobiluncus obtiveram maior representatividade, seguida por Trichomonas vaginalis. Estes resultados se assemelham a estudo realizado no município de João Pessoa-Paraíba em 2006, em que $13,1 \%$ dos resultados apresentaram Cândida sp., 3,14\% Gardnerella/Mobiluncus e 0,66\% Trichomonas vaginalis ${ }^{(16)}$. Desta forma, observa-se a necessidade do profissional de saúde identificar, junto à paciente, quais fatores estão relacionados a esses achados, possibilitando a implementação de medidas que visem à diminuição de infecção por esses agentes.

Os epitélios mais prevalentes foram o escamoso e o glandular e, em menor representatividade, o metaplásico. Observou-se, deste modo, que os epitélios identificados em maior proporção fazem parte da fisiologia feminina, e seu crescimento é hormônio-dependente, podendo alterar-se com o ciclo menstrual, correlacionando o seu equilíbrio ou desequilíbrio. A presença do tecido metaplásico é indicativo de diferenciação no epitélio estas células estão mais sujeitas a fatores, provavelmente múltiplos, relacionados à gênese do carcinoma escamoso do colo uterino. Deve-se dar maior atenção a esse achado, principalmente nas mulheres que se encontram em idade reprodutiva ${ }^{(17)}$.

Ressalta-se que devido às altas taxa de mortalidade causada pelo câncer do colo uterino, o mesmo deve ser compreendido como um problema de saúde pública de grande relevância, o que sinaliza a importância de ações para sua prevenção e detecção precoce ${ }^{(6)}$.

\section{CONCLUSÃO}

Este estudo esteve associado à prevalência do registro de laudos de exame de Papanicolaou realizados, predominante em mulheres em idade reprodutiva, suscitando pesquisas associadas à motivação para a realização do citado exame, que poderia ter sua busca relacionada à presença de alterações na microbiota vaginal, como observado na caracterização dos laudos citopatológicos verificados.

Identificou-se a necessidade de fortalecer a realização do exame preventivo em mulheres que se distanciam da idade reprodutiva, buscando-se incentivá-las à adesão para a realização periódica do exame de Papanicolaou. Esta conduta permite o acompanhamento das possíveis afecções ginecológicas e melhoria na resolutividade dessas. Destarte, a realização periódica do exame de Papanicolaou perpassa pelas estratégias adotadas pelos serviços de saúde para estimulo à adesão e ao seguimento assistencial, conforme o laudo do exame de Papanicolaou, fundamentadas na busca ativa e em ações de educação em saúde, favorecendo a promoção de sua saúde.

Por outro lado, o registro significativo de Gardnerella/Mobiluncus nos laudos de Papanicolaou evidencia a necessidade do profissional de saúde identificar quais os fatores relacionados a esse achado e assim promover o bem estar da mulher, adotando a conduta clínica apropriada. A presença de Trichomonas vaginalis, mesmo em número relativamente baixo, mostra a necessidade de identificação de fatores de risco e aconselhamento em relação à proteção, transmissão e tratamento dessa DST, como também para outras patologias desse grupo, apontando para a disseminação das informações necessárias para o controle de DST, e ao envolvimento do parceiro sexual.

Por fim, este estudo limitou-se quanto às informações adicionais referentes ao perfil sociodemográfico das mulheres que realizaram os exames de Papanicolaou devido a falta do registro dessas informações na fonte consultada.

\section{REFERÊNCIAS}

1. Ministério da Saúde (BR). Secretaria de Atenção à Saúde. Departamento de Atenção Básica. Controle dos cânceres do útero e da mama. Brasília: Ministério da Saúde; 2006.

2. World Health Organization. International Agency for Research on Cancer. Globocan 2008. Lyon, 2008. [Internet] 2009 [acesso em 30 jul. 2012]. Disponível: http://globocan.iarc.fr/>.

3. Instituto Nacional de Câncer (INCA). Estimativa 2012. Incidência do câncer no Brasil. Rio de Janeiro: INCA, 2011.

4. Pereira JC, Ganassin FMH, Oliveira RD, Renovato RD, Watanabe EAMT. Morbidade por câncer do colo uterino em mulheres da reserva indígena no Mato Grosso do Sul. Cogitare enferm. [Internet] 2011;16(1) [acesso em 30 jul 2012]. Disponível: http://ojs.c3sl.ufpr.br/ojs2/ index.php/cogitare/article/viewArticle/21123

5. Spitzer M, Krumholz BA. Human papilomavirusrelated deseases in the female patient. Urol Clin N Am. 
[Internet] 1992;19(1) [acesso em 30 jul 2012]. Disponível: http://www.ncbi.nlm.nih.gov/pubmed/1310548

6. Pinho AA, França-Júnior I. Prevenção do câncer de colo do útero: um modelo teórico para analisar o acesso e a utilização do teste de Papanicolaou. Rev. Bras. Saúde Matern. Infant. [Internet] 2003; 3(1) [acesso em 30 jul 2012]. Disponível: http://www. scielo.br/scielo.php?script=sci_arttext\&pid=S1519$38292003000100012 \& \operatorname{lng}=$ pt\&nrm=iso ISSN 1519-3829. http://dx.doi.org/10.1590/S151938292003000100012

7. Oliveira SL, Almeida ACH. A percepção das mulheres frente ao exame de papanicolaou: da observação ao entendimento. Cogitare enferm. [Internet] 2009;14(3) [acesso em 30 jul. 2012]. Disponível: http:/ojs.c3sl.ufpr. br/ojs2/index.php/cogitare/article/viewArticle/16183

8. Stuczynsky JV, Naud P, Matos JC. Vulvovaginites e cervicites. In: Pasqualotto AC, Schwarzbold AV, organizadores. Doenças infecciosas. Porto Alegre: Artmed; 2006. p. 342-44.

9. Silva MCP, Dimech GS, Silva AJF, Amaral LBC, Gonçalves ES. Avaliação do perfil epidemiológico das pacientes atendidas no núcleo de saúde da UFRPE portadoras de Vlvovaginites. In: IV Jornada de Ensino, Pesquisa e Extensão - JEPEX; 2009 out. p. 19-23; Pernambuco, Brasil. Pernambuco: UFRPE; 2009.

10. Martins MCL, Bôer CG, Svidzinsk TIE, Donida LG, Martins PFA, Boscoli FNS, et al. Avaliação do método de Papanicolaou para triagem de algumas infecções cérvico-vaginais. Rev. bras. anal. clin. [Internet] 2007;39(3) [acesso em 30 jul 2012]. Disponível: http://www.sbac.org.br/pt/pdfs/rbac/rbac_39_03/ rbac_39_3_13.pdf

11. Ribeiro AA, Oliveira DF, Sampaio MCN, Carneiro MAS, Tavares SBN, Souza NLA, et al. Agentes microbiológicos em exames citopatólogicos: estudo de prevalência. Rev. bras. anal. clin. [Internet] 2007;39(3) [acesso em 30 jul 2012]. Disponível: https://sbac.org.br/ $\mathrm{pt} / \mathrm{pdfs} / \mathrm{rbac} / \mathrm{rbac}$ 39_03/rbac_39_3_05.pdf

12. Gil AC. Como elaborar projetos de pesquisa. $4^{\mathrm{a}} \mathrm{ed}$. São Paulo: Atlas; 2006.

13. Hoyo C, Miller WC, Newman BM, Fortney JA. Selective screening for cervical neoplasia: an approach for resource-poor settings. Int. J. Epidemiol. [Internet] 2000;29(5) [acesso em 30 jul 2012]. Disponível: http://ije. oxfordjournals.org/content/29/5/807.short
14. Bernard VB, Eheman CR, Lawson HW, Blackman DK, Anderson C, Helsel W, et al. Cervical screening in the national breast and cervical cancer early detection program, 1995-2001. Obstet Gynecol. [Internet] 2004;103(3) [acesso em 30 jul 2012]. Disponível: http://www.ncbi.nlm.nih.gov/ pubmed/14990422

15. Linhares IM, Giraldo PC, Baracat EC. Novos conhecimentos sobre a flora bacteriana vaginal. Rev. Assoc. Med. Bras. [Internet] 2010;56(3) [acesso em 30 jul 2012]. Disponível: http://www.scielo.br/pdf/ramb/ v56n3/v56n3a26.pdf

16. Santana AP, Gomes AC, Marques KJF, Andrade KKP, Silva FMC, Gomes JS. Afecções ginecológicas evidenciadas no Papanicolaou em uma unidade de saúde da família na cidade de João Pessoa. In: X Encontro de Extensão da UFPB; 2006 ago. p.1-9; Paraíba, Brasil. Paraíba: UFPB; 2006.

17. Araújo SR. Citologia e histologia básica do colo uterino para ginecologistas: uma sessão de slides. Curitiba: VB; 1999. 